\title{
Árvores frutíferas nos quintais urbanos de Boa Vista, Roraima, Amazônia brasileira
}

\author{
Rui Jorge da Conceição Gomes SEMEDOํ, Reinaldo ImbrozioBARBOSA²
}

\section{RESUMO}

O objetivo do trabalho foi estimar a riqueza e a diversidade das espécies de árvores frutíferas cultivadas nos quintais caseiros da cidade de Boa Vista, Roraima, bem como determinar quais são as espécies cultivadas preferencialmente pela população urbana local. Os levantamentos foram realizados em dois bairros surgidos com a expansão da cidade em 1982: (1) BEst Bairro dos Estados (Zona Norte) e (2) BAsa - Bairro Asa Branca (Zona Oeste). Foram observados 722 quintais no BEst (06 a 22.03.2004) e 339 no BAsa (07.04 a 01.07.2004). Trinta e seis espécies (19 famílias botânicas) foram encontradas no BEst e 37 (20 famílias) no BAsa, configurando um total de 43 espécies (20 famílias) observadas. Deste total, 30 espécies (69,8\%) de 19 famílias (95\%) ocorreram em ambos os bairros, sugerindo preferências frutíferas comuns. Os três maiores índices de valor de preferência (IVP) foram coincidentes e registrados para coco (Cocos nucifera L. - BEst: 19,4\% e BAsa: 20,5\%), manga (Mangifera indica L. - BEst: 14,9\% e BAsa: 22,5\%) e jambo (Syzygium malaccence (L.) Merr. \& L.M. Perry - BEst: 10,5\% e BAsa: 10,1\%), todos de origem externa à Amazônia, mas que congregaram conjuntamente 44,9\% (BEst) e 53,0\% (BAsa) de IVP. Estes resultados sugerem que o cultivo de árvores frutíferas em quintais caseiros de Boa Vista segue um padrão que concentra a escolha em poucas espécies, não-originárias da Amazônia, mas tradicionalmente consagradas por seu êxito na produção de frutos.

PALAVRAS-CHAVE: Amazônia, Frutas, Pomar caseiro, Diversidade.

\section{Fruit trees in urban home gardens of Boa Vista, Roraima, Brazilian Amazonia}

\begin{abstract}
The objective of this study was to estimate the richness and the diversity of fruit tree species cultivated in Boa Vista's home gardens, as well as to determine what species the local urban population prefers. Two neighborhoods that originated during the city's expansion in 1982 were sampled: (1) BEst - Bairro dos Estados (North Zone) and (2) BAsa - Bairro Asa Branca (West Zone). Seven hundred and twenty-two home gardens were surveyed in BEst (March 6 to 22, 2004), and 339 in BAsa (April 7 to July 1, 2004). Thirty-six species (19 botanical families) were observed in BEst, and 37 (20 families) in BAsa, totaling 43 species (20 families). Thirty species (69.8\%) in 19 families (95\%) occurred in both neighborhoods, suggesting common fruit preferences. The three largest indices of preference value (IVP) were coconut (Cocos nucifera L. - BEst: 19.4\% and BAsa: 20.5\%), mango (Mangifera indica L. - BEst: $14.9 \%$ and BAsa: 22.5\%) and rose-apple (Syzygium malaccence (L.) Merr. \& L.M. Perry - BEst: $10.5 \%$ and BAsa: 10.1\%). All of them are exotic (originating in Southeast Asia) and together had IVPs of $44.9 \%$ (BEst) and $53.0 \%$ (BAsa). These results suggest that the cultivation of fruit trees in the home gardens of Boa Vista shows a pattern that concentrates choices in a few non-Amazonian species that are traditionally preferred because of their successful fruit production.
\end{abstract}

KEYWORDS: Amazonia, Fruits, Home garden, Diversity.

\footnotetext{
1 Universidade Federal de Roraima, Centro de Ciências Sociais e Geociências, Coordenação de Ciências Sociais. Avenida Venezuela sn - Jardim Floresta. CEP 69300-000. Boa Vista RR. Telefone: (95) 6213160. e-mail: rjogos18@yahoo.com.br

2 e-mail: reinaldo@inpa.gov.br
} 


\section{INTRODUÇÃO}

Quintais associados a habitações humanas podem ser classificados como sistemas agroflorestais funcionando como reservatórios de diversidade de espécies de árvores, arbustos e ervas situados dentro de um limite residencial, e sob o manejo e o trabalho familiar (Fernandes \& Nair, 1986; McConnell, 1992; Nair, 1993). Outros termos podem ser utilizados para este espaço como "home garden", sítio, pomar caseiro ou terreiro (Martins et al., 2003). Do ponto de vista cultural, a reunião de plantas e animais próximos de habitaçôes humanas pode revelar muito da história cultural dos lugares e das decisões de manejo dos proprietários individuais (Blanckaert et al., 2004). De forma geral, podem satisfazer alguns requerimentos básicos de alimentação, fibras, remédios, construção, recreação e experiências estéticas (Kimber, 1966 citado em WinklerPrins, 2002), além de remover carbono da atmosfera estocando-o na forma de biomassa terrestre (Albrecht \& Kandji, 2003).

$\mathrm{Na}$ Amazônia, os quintais residenciais são de grande importância tanto na vida rural como na urbana, quase sempre expressando um "contínuo rural-urbano" (WinklerPrins, 2002). Este "contínuo" representa um intercâmbio de material genético que associa a diversidade contida nos ecossistemas naturais às tradiçôes das populações humanas locais (Balée, 1989; Clement et al., 2001), alcançando fortemente a vida urbana através da abertura de espaço na complementação alimentar dos habitantes das cidades regionais (Martins, 1998). Estudos que atentem para o entendimento do aproveitamento deste espaço como forma de subsídio de parte da vida urbana atual são ainda escassos e de pouca expressão na Amazônia. Entretanto, a FAO (Food and Agriculture Organization of the United Nations) vem atentando que os "... pomares caseiros estão também se transformando em uma importante fonte de alimento e renda para propriedades pobres de áreas urbanas $e$ peri-urbanas" (FAO, 2004), podendo mitigar parcialmente a falta de alimentos e nutrientes em populações humanas destes centros.

Dos grupos de espécies botânicas utilizadas por populaçōes sedentárias na forma de quintais caseiros na Amazônia, o de maior expressão, ou pelo menos o de maior visibilidade, é o da categoria "perene" (ver Clement, 1999a para o contexto histórico). Neste grupo se encaixam as árvores frutíferas comestíveis que incorporam à alimentação diferentes fontes de vitaminas, de suma importância ao metabolismo fisiológico humano, podendo também oferecer sombra e lazer, além de se enquadrarem no grupo das medicinais alternativas (MS, 2002; Madaleno, 2000).

$\mathrm{Na}$ região amazônica, as adaptações das populaçōes humanas (tradicionais ou externas à região) em ambientes urbanos podem acarretar diferenças no uso destes recursos (Martins, 1998). Fatores como migração, por exemplo, incorporam uma bagagem cultural externa no uso dos recursos regionais, fazendo com que variedades não-existentes na localidade (exóticas) sejam incorporadas e/ou maximizadas aos hábitos locais. No sentido de iniciar um entendimento de como são estes padróes de uso dos recursos em um ambiente urbano amazônico, este estudo de caso teve por objetivo estimar a riqueza e a diversidade de espécies de árvores frutíferas cultivadas nos quintais caseiros da cidade de Boa Vista, Roraima, bem como o padrão de preferência do cultivo destas espécies pela população urbana local.

\section{MATERIAL E MÉTODOS}

\section{ÁREA DE ESTUDO}

Boa Vista é a capital do estado de Roraima, situada no setor centro-sul do maior bloco contínuo de savanas do extremo norte da Amazônia brasileira (Sanaiotti, 1996; Miranda, 1998). Para cumprir com o objetivo do estudo foi realizada uma amostragem rápida em dois bairros criados com a expansão da cidade em 1982: (1) BAsa - Bairro Asa Branca (Zona Oeste) e (2) BEst - Bairro dos Estados (Zona Norte). O critério de escolha dos bairros foi o de excluir aqueles criados recentemente $(<5$ anos), por que os recursos amostrados ainda não estariam facilmente visíveis e em plena produção, e bairros muito antigos do centro da cidade e adjacências $(>40$ anos), por estarem concentrados nas áreas de prédios públicos e comerciais, com maior investimento em estrutura física do que em quintais frutíferos.

Os dois bairros escolhidos incorporam renda mensal média familiar diferentes $(\mathrm{BAsa}=\mathrm{R} \$ 603,00$ e BEst $=\mathrm{R} \$$ $1.053,00$ ), considerado o ano de 2000 (IBGE, 2004), mas são quase que exclusivamente residenciais, representando o objetivo deste trabalho. Eles foram criados para suprir não só a demanda de migrantes chegados em Roraima no início dos anos 1980, como também de habitantes locais que se deslocaram de antigos redutos rurais (pecuários e minerais) do interior do Estado. Em ambos os casos, a área útil de cada lote urbano varia de 415 a $600 \mathrm{~m}^{2}$. A população total presente nestes bairros foi contabilizada em 4.560 pessoas para o BEst e 10.017 para o BAsa (IBGE, 2004). A decisão de concentrar esforços em apenas dois bairros foi a de aumentar o número de casas observadas, devido à facilidade de coleta de dados, partindo da hipótese de que a distribuição das diferentes correntes migratórias seria eqüitativa ao longo de uma grande amostragem, independente de renda familiar média mensal ou da origem das famílias. Como a estrutura da origem e da procedência dos imigrantes não foi alterada significativamente nestes últimos anos (IBGE, 2002), nós assumimos que os dois bairros possam inferir uma situação média migratória da realidade local atual.

\section{COLETA DE DADOS}

O método empregado neste trabalho foi baseado em um inventário rápido através da observação externa de cada 
unidade ou módulo familiar (casa urbana), permitindo amostrar um grande número de quintais em pouco tempo de atividade de campo. As árvores frutíferas são facilmente visíveis e permitem poucos erros na contabilização ou identificação externa, mesmo considerando muros ou outros obstáculos que dificultem a visão de espécies/indivíduos de menor porte.

O número de unidades observadas no BEst foi de 722 residências $(53,9 \%$ do total existente) no período de 06 a 22.03.2004. As ruas amostradas, a partir de uma escolha aleatória inicial, foram sempre alternadas para tentar obter maior representatividade dentro do bairro. Todas as casas das ruas contempladas foram observadas e contabilizadas. Residências sem árvores frutíferas e terrenos abandonados também foram contabilizados. Em seguida, foram amostradas 339 unidades no BAsa (10,5\% do total), entre 07.04 e 01.07.2004. O BAsa sofreu uma intensidade amostral menor porque as observações realizadas no BEst indicaram que o levantamento de $10 \%$ das residências presentes refletiria $\approx 95 \%$ do total de espécies do bairro, sendo considerado representativo.

Foram anotados as espécies arbóreo-frutíferas e o número de indivíduos que cada espécie comportava no espaço destinado de cada residência observada. A definição de árvore frutífera neste estudo compreende dois sentidos: amplo, incluindo todas as plantas de porte arbóreo (ou similar) pertencentes aos grupos das Angiospermas e das Gimnospermas e, restrito, implicando que ela deva ser tradicionalmente comestível pela população humana. Foram também consideradas as espécies e os indivíduos sob domínio da residência, como aquelas presentes nas calçadas. Apenas os indivíduos com porte visível de produção (adultos) foram contabilizados, descartando-se aqueles na fase juvenil. Espécies com dúvidas na identificação pelo observador foram selecionadas para que o proprietário se manifestasse quanto ao nome vulgar e/ou a possibilidade de coleta de material fértil para posterior identificação botânica. A maioria das identificações taxonômicas foi feita através de conhecimento prévio dos autores, e de consulta à literatura (Silva et al., 1977; Pahlen et al., 1979; Rodrigues, 1989; Cavalcante, 1991; Shanley et al., 1998; Miranda et al., 2001), visto que quase todas as plantas observadas são de uso comum e sem dificuldades de identificação. Os nomes científicos das plantas e dos autores foram checados no portal do Missouri Botanical Garden (www.mobot.org). Plantas com dúvidas na identificação científica foram classificadas apenas até gênero.

\section{ANÁLISE DOS DADOS}

Todos os dados foram tabelados e analisados segundo a riqueza (número de espécies, S) e a diversidade (Índice de Shannon-Weiner, H' - base 10) das espécies de árvores frutíferas por bairro. A similaridade do conjunto das espécies encontradas nos dois bairros foi obtida através do Índice de Sørensen para testar a hipótese de que, independente da situação sócio-econômica, ambos representam de forma mediana a riqueza e a diversidade de espécies arbóreofrutíferas de Boa Vista. As fórmulas e a sequiência de cálculos dos parâmetros supracitados foram adaptadas de Magurran (1988) e Kent \& Coker (1994). A provável origem (centro de dispersão) de cada espécie também foi tabelada a partir das informaçōes contidas nas mesmas literaturas utilizadas para consulta de nomes.

Um índice de valor de preferência (IVP) foi gerado para identificar o padrão de uso das principais espécies plantadas nos quintais de Boa Vista. Este índice é a soma relativa (\%) da abundância (número de indivíduos observados por espécie) e da freqüência (número de residências em que a espécie aparece) das espécies em cada bairro. Ele é calculado da seguinte forma:

$$
\mathrm{IVP} \%=\mathrm{Abu}-\mathrm{Re} \mathrm{l} \%+\text { Fre-Rel } \%
$$

Onde Abu-Rel\% = número de indivíduos de uma espécie, dividido pelo número total de indivíduos de todas as espécies observadas em cada bairro, multiplicado por 100; e Fre-Rel\% = número de residências em que uma determinada espécie ocorre, dividido pelo número total de residências observadas, multiplicado por 100 .

\section{RESULTADOS}

Foram observados 2.534 indivíduos distribuídos em 36 espécies e 19 famílias botânicas no Bairro dos Estados (BEst), e 2.167 indivíduos de 37 espécies (20 famílias) no Bairro Asa Branca (BAsa) (Tabela 1). Do total de 43 espécies observadas, $30(69,8 \%)$ de 19 famílias $(95,0 \%)$ são comuns em ambos os bairros. Os três maiores índices de valor de preferência (IVP) das espécies foram coincidentes e registrados para coco (Cocos nucifera L. - BEst: 19,4\% e BAsa: 20,5\%), manga (Mangifera indica L. - BEst: 14,9\% e BAsa: 22,5\%) e jambo (Syzygium malaccence (L.) Merr. \& L.M. Perry - BEst: 10,5\% e BAsa: $10,1 \%)$, todos de origem externa à Amazônia, mas que congregaram conjuntamente $44,9 \%$ (BEst) e $53,0 \%$ (BAsa) de IVP. As famílias com maior concentração de indivíduos foram Anacardiaceae (BEst: 20,8\% e BAsa: 30,5\%), Arecaceae (BEst: 23,0\% e BAsa: 23,9\%) e Myrtaceae (BEst: 18,5\% e BAsa: $14,9 \%)$. De todas as espécies observadas, 23 são originárias dos trópicos americanos, sendo nove exclusivas da região amazônica. $\mathrm{O}$ índice de diversidade de Shannon $(\mathrm{H})$ foi de 1,11 para o BEst e pouco inferior $(1,04)$ para o BAsa. A similaridade (Sørensen) do conjunto das espécies entre os dois bairros foi de 0,82 .

Levando em consideração todos as residências observadas, inclusive as abandonadas e as sem nenhum tipo de quintal arbóreo-frutífero, a média do número de indivíduos por 
Tabela 1 - Espécies de árvores frutíferas (abundância, freqüência e IVP) identificadas nos quintais urbanos de Boa Vista, Roraima.

\begin{tabular}{|c|c|c|c|c|c|c|c|c|c|c|}
\hline \multirow[b]{2}{*}{ Família } & \multirow[b]{2}{*}{ Nome Científico } & \multirow{2}{*}{ 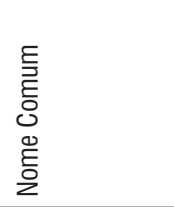 } & \multirow{2}{*}{ 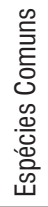 } & \multirow{2}{*}{$\frac{\text { 岕 }}{\text { 㟧 }}$} & \multirow{2}{*}{ 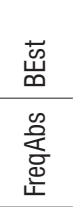 } & \multirow{2}{*}{ 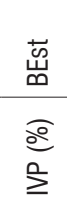 } & \multirow{2}{*}{ 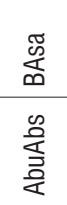 } & \multirow{2}{*}{ 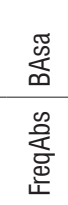 } & \multirow{2}{*}{ 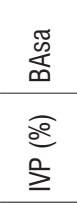 } & \multirow[b]{2}{*}{ Origem (provável) } \\
\hline & & & & & & & & & & \\
\hline \multirow[t]{4}{*}{ Anacardiaceae } & Anacardium occidentale L. (1a) & Caju & $\mathrm{x}$ & 136 & 108 & 5,7 & 129 & 82 & 6,4 & Litoral Atlântico da América Tropical \\
\hline & Mangifera indica L. (1b) & Manga & $x$ & 386 & 262 & 14,9 & 526 & 249 & 22,5 & Sul da Ásia \\
\hline & Spondias mombim L. & Taperebá & & - & 0 & 0,0 & 1 & 1 & 0,06 & America do Sul e Central \\
\hline & Spondias purpurea L. & Siriguela & $\mathrm{x}$ & 5 & 5 & 0,2 & 2 & 2 & 0,13 & Trópicos americanos \\
\hline \multirow[t]{5}{*}{ Annonaceae } & Annona muricata L. & Graviola & $x$ & 6 & 6 & 0,3 & 7 & 6 & 0,4 & Trópicos americanos \\
\hline & Annona squamosa L. & Ata & $x$ & 97 & 72 & 3,9 & 53 & 33 & 2,6 & provavelmente Antilhas \\
\hline & Annona cf montana Macf. & Araticum & & 1 & 1 & 0,05 & - & 0 & 0,0 & Antilhas ou Amazônia (?) \\
\hline & A. cherimolia Mill. xA. squamosa L. & Atemoia & & 1 & 1 & 0,05 & - & 0 & 0,0 & Híbrido (trópicos americanos) \\
\hline & Rollinia mucosa (Jacq.) Bail. & Biribá & & - & 0 & 0,0 & 1 & 1 & 0,06 & Amazônia \\
\hline \multirow[t]{5}{*}{ Arecaceae } & Astrocaryum aculeatum G. F. W. Meyer & Tucumã & & 5 & 4 & 0,2 & - & 0 & 0,0 & Norte da América do Sul \\
\hline & Bactris gasipaes Kunth. & Pupunha & $x$ & 4 & 2 & 0,1 & 6 & 5 & 0,3 & Trópicos americanos \\
\hline & Cocos nucifera L. (1c) & Coco & $x$ & 553 & 306 & 19,4 & 499 & 217 & 20,5 & Litorais tropicais do oceano Atlântico \\
\hline & Euterpe oleraceae Martius & Açai & $x$ & 8 & 4 & 0,3 & 6 & 4 & 0,3 & Amazônia \\
\hline & Mauritia flexuosa L. & Buriti & $x$ & 14 & 13 & 0,6 & 7 & 6 & 0,4 & Amazônia \\
\hline Caesalpinaceae & Tamarindus indica L. & Tamarindo & $x$ & 9 & 6 & 0,3 & 5 & 4 & 0,3 & Índia \\
\hline Caricaceae & Carica papaya L. (1d) & Mamão & $x$ & 144 & 100 & 5,6 & 58 & 39 & 3,0 & Trópicos americanos \\
\hline Lauraceae & $\begin{array}{l}\text { Persea americana Mill. var } \\
\text { americana }\end{array}$ & Abacate & $\mathrm{x}$ & 181 & 153 & 7,8 & 98 & 71 & 5,2 & $\begin{array}{l}\text { América Central e Norte da América } \\
\text { do Sul }\end{array}$ \\
\hline \multirow[t]{2}{*}{ Malpighiaceae } & Byrsonima sp. & Mirixi & $x$ & 3 & 3 & 0,14 & 4 & 3 & 0,2 & Trópicos americanos \\
\hline & Malpighia punicifolia L. & Acerola & $\mathrm{x}$ & 195 & 155 & 8,2 & 165 & 97 & 7,8 & $\begin{array}{l}\text { América do Sul, Antilhas e América } \\
\text { Central }\end{array}$ \\
\hline Mimosaceae & Inga cf edulis Mart. & Ingá & $x$ & 2 & 2 & 0,10 & 11 & 11 & 0,7 & Trópicos e sub-trópicos americanos \\
\hline \multirow[t]{2}{*}{ Moraceae } & $\begin{array}{l}\text { Artocarpus altulis (Sol. ex. Park) } \\
\text { Fosb. }\end{array}$ & Fruta Pão & & 1 & 1 & 0,05 & - & 0 & 0,0 & Sul do Pacífco (Java e Sumatra) \\
\hline & Artocarpus heterophyllus Lam. & Jaca & $x$ & 4 & 4 & 0,2 & 10 & 8 & 0,6 & Índia \\
\hline Musaceae & Musa spp. & Banana & $x$ & 48 & 33 & 1,9 & 62 & 19 & 2,2 & Ásia \\
\hline \multirow[t]{5}{*}{ Myrtaceae } & Eugenia cumini (L.) Druce & Azeitona Roxa & $x$ & 1 & 1 & 0,05 & 2 & 2 & 0,13 & Índia \\
\hline & Eugenia uniflora L. & Pitanga & & - & 0 & 0,0 & 4 & 4 & 0,3 & Brasil \\
\hline & Psidium guajava L. (1e) & Goiaba & $x$ & 174 & 143 & 7,4 & 91 & 63 & 4,7 & Trópicos americanos \\
\hline & Psidium guianense Pers. & Araçá & $x$ & 4 & 4 & 0,2 & 3 & 2 & 0,2 & México até Argentina \\
\hline & $\begin{array}{l}\text { Syzygium malaccence (L.) } \\
\text { Merr.\&L.M.Perry }\end{array}$ & Jambo & $\mathrm{x}$ & 291 & 171 & 10,5 & 224 & 118 & 10,1 & Malásia \\
\hline Oxalidaceae & Averrhoa carambola L. & Carambola & $x$ & 30 & 27 & 1,3 & 33 & 31 & 2,0 & Ásia Tropical \\
\hline Punicaceae & Punica granatum L. & Romã & $x$ & 19 & 17 & 0,8 & 7 & 7 & 0,5 & Ásia \\
\hline Rhamnaceae & Zizyphus mauritiana Lam. & Dão & $x$ & 9 & 7 & 0,4 & 3 & 3 & 0,2 & Sul da Ásia \\
\hline Rosaceae & Rubus urticaefolius Sairet. & Amora & & - & 0 & 0,0 & 4 & 4 & 0,3 & Europa \\
\hline Rubiaceae & Genipa americana L. & Jenipapo & $\mathrm{x}$ & 9 & 7 & 0,4 & 1 & 1 & 0,06 & Norte da América do Sul \\
\hline \multirow[t]{5}{*}{ Rutaceae } & $\begin{array}{l}\text { Citrus aurantifolia (Christm.) } \\
\text { Swingle }\end{array}$ & Limão Galego & & 1 & 1 & 0,0 & - & 0 & 0,0 & Ásia \\
\hline & Citrus limonia (L.) Osbeck & Limão & $x$ & 66 & 59 & 2,9 & 90 & 67 & 4,9 & Ásia \\
\hline & Citrus medica L. & Lima (Cidra) & & - & 0 & 0,0 & 2 & 2 & 0,13 & Ásia \\
\hline & Citrus nobilis Lour. var. deliciosa & Tangerina & & - & 0 & 0,0 & 1 & 1 & 0,06 & Ásia \\
\hline & Citrus sinensis (L.) Osbeck & Laranja & $x$ & 29 & 27 & 1,3 & 8 & 8 & 0,5 & Ásia \\
\hline Sapindaceae & Talissia esculenta (St. Hil.) Radlk & Pitomba & $x$ & 34 & 33 & 1,6 & 12 & 10 & 0,7 & Oeste da Amazônia \\
\hline \multirow[t]{2}{*}{ Sapotaceae } & Pouteria sp1 & Abiurana & & 4 & 3 & 0,2 & - & 0 & 0,0 & Amazônia \\
\hline & Pouteria cf caimito Radlk. & Abiu & & - & 0 & 0,0 & 4 & 4 & 0,3 & Amazônia \\
\hline
\end{tabular}


Tabela 1 - Continuação

\begin{tabular}{|c|c|c|c|c|c|c|c|c|c|c|}
\hline \multirow[b]{2}{*}{ Família } & \multirow[b]{2}{*}{ Nome Científico } & \multirow{2}{*}{ 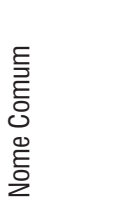 } & \multirow{2}{*}{ 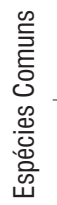 } & 岕 & $\begin{array}{l}\text { 岕 } \\
\text { 品 }\end{array}$ & 岕 & 总 & 总 & 芯 & \multirow[b]{2}{*}{ Origem (provável) } \\
\hline & & & & $\begin{array}{l}\text { o } \\
\text { 这 } \\
\text { 远 }\end{array}$ & 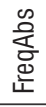 & $\begin{array}{l}\widehat{o} \\
\stackrel{\varrho}{\geq}\end{array}$ & $\begin{array}{l}\text { 足 } \\
\text { 竞 }\end{array}$ & 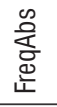 & $\begin{array}{l}\text { o } \\
\geq\end{array}$ & \\
\hline \multirow[t]{2}{*}{ Sterculiaceae } & Theobroma cacao L. & Cacau & $x$ & 1 & 1 & 0,05 & 9 & 9 & 0,6 & Amazônia \\
\hline & $\begin{array}{l}\text { T. grandiflorum (Willd. ex. } \\
\text { Spreng) Schum. }\end{array}$ & Cupuaçu & $x$ & 59 & 52 & 2,6 & 19 & 12 & 0,9 & Amazônia \\
\hline \multicolumn{2}{|l|}{$\begin{array}{l}\text { Total de } \\
\text { Indivíduos }\end{array}$} & & \multicolumn{3}{|c|}{2534} & \multicolumn{3}{|c|}{2167} & & \\
\hline \multicolumn{2}{|l|}{ Riqueza (S) } & & \multicolumn{3}{|c|}{36} & \multicolumn{3}{|c|}{37} & & \\
\hline \multicolumn{2}{|c|}{ Diversidade (Shannon - H) } & & \multicolumn{2}{|r|}{1,11} & & \multicolumn{3}{|c|}{1,04} & & \\
\hline
\end{tabular}

(1) Número de variedades identificadas: (a) duas (amarela e vermelha), (b) quatro (manquita, espada, rosa e coracão-de-boi), (c) três (anão, verde-gigante e amarelo), (d) duas (papaia e regional), (e) duas (branca e vermelha); (2) AbuAbs é a abundância absoluta e FreAbs é a frequêencia absoluta das espécies. 0 IVP é a soma da freqüência e da abundância na forma relativa (\%).

residência foi de 3,4 $\pm 2,9$ (DP) (BEst) e de 6,8 \pm 4,2 (BAsa), enquanto que o número médio de espécies por residência foi de 2,4 $\pm 1,8$ (BEst) e de 3,8 $\pm 2,0$ (BAsa) (Figura 1). Ambos os casos diferiram significativamente no nível de $5 \%\left(\mathrm{t}_{0,05(2)}\right)$
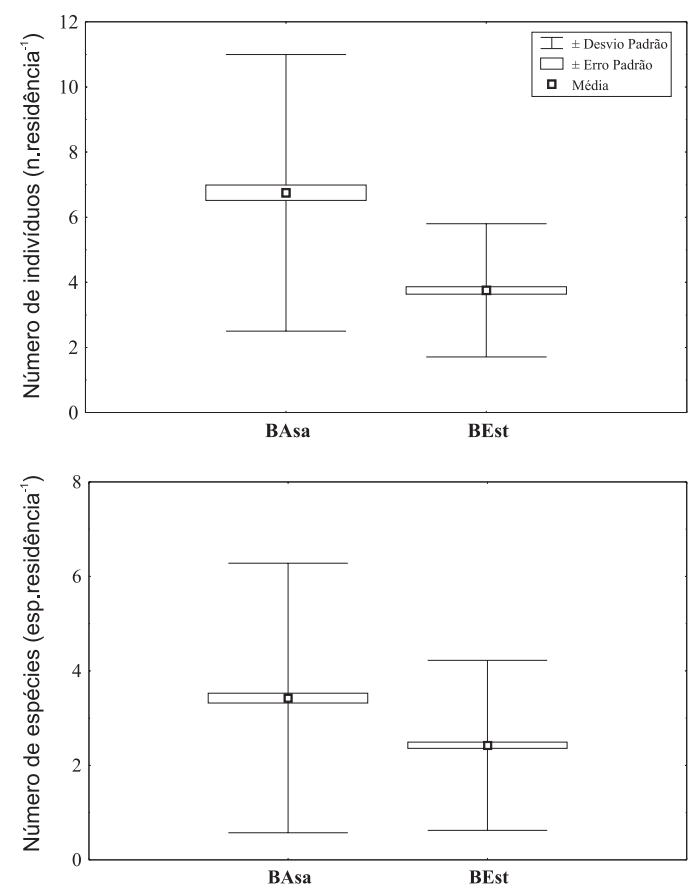

Figura 1 - Número médio de indivíduos (n.residência-1) e espécies (esp. residência-1) de árvores frutíferas presentes nos quintais amostrados dos bairros Asa Branca (BAsa) e dos Estados (BEst), na cidade de Boa Vista, Roraima.

\section{DISCUSSÃO}

Existe baixa diversificação e forte concentração de indivíduos em poucas espécies (Índice de Shannon $\approx 1$ ) nesses bairros de Boa Vista, caracterizando um acentuado investimento naquelas tradicionalmente conhecidas. Embora haja 43 espécies de árvores frutíferas difundidas por ambos os bairros, apenas três delas congregam aproximadamente $50 \%$ da preferência de cultivo nas residências. Os hábitos de escolha geral das espécies nos dois bairros sugerem uma alta correspondência, comprovado pelo índice de similaridade de Sørensen $(0,82)$, indicando não haver grandes diferenças preferenciais entre bairros, sendo 30 espécies comuns aos dois. Isto corroboraria a hipótese de que existem hábitos preferenciais similares para a escolha das principais espécies fruti-arbóreas utilizadas pela população urbana local, embora a quantidade de indivíduos e o número de espécies utilizadas tenham diferido entre os bairros avaliados. Isto ocorre porque as espécies comuns aos dois bairros são as que possuem os maiores índices de valor de preferência.

O grau de importância deste tipo de recurso urbano em Boa Vista para as residências familiares sugere estar associado diretamente à renda da população residente nestes bairros. No bairro de renda familiar mensal maior (BEst) o número médio de espécies arbóreo-frutíferas por residência foi $37 \%$ inferior ao do BAsa, enquanto o número de indivíduos presentes neste último foi o dobro do encontrado no BEst. A distribuição do número médio de indivíduos por residência também foi mais equilibrada no BAsa do que no BEst, que concentrou o maior número de residências (41\%) com menor quantidade de indivíduos (1 a 3 por quintal). Embora carente de um estudo mais aprofundado que envolva conhecimento tradicional em ambas as comunidades, como sugerido por Martin (1995), nós observamos que o bairro de maior renda (BEst) optou em investir mais em bens materiais (área construída) e segurança (muros, cercas, etc.) do que no cultivo de árvores frutíferas, que tomam muito espaço físico. Entretanto, o de menor renda (BAsa) sugere um aproveitamento mais intenso deste tipo de recurso como fonte suplementar nutricional (consumo familiar) e/ou laser. O comércio de pequeno escala deve ser uma alternativa local presente, mas muito pouco se sabe sobre a importância deste fator na renda familiar das cidades. 
A suplementação alimentar pode não ser explicitamente intencional neste caso, mas a diversificação produz melhores efeitos nutricionais do que o cultivo de uma grande quantidade de uma ou duas espécies. Intencional ou não, a maioria das frutas cultivadas nos quintais de Boa Vista é rica em vitamina C (Tabela 2), com concentração acima de $9 \mathrm{mg} \cdot 100 \mathrm{~g}^{-1}$ (MS, 2000), como acerola, caju e goiaba que estão entre as mais cultivadas nos quintais. Entretanto, o conjunto das frutíferas observadas é pobre em vitamina $\mathrm{A}\left(<120 \mathrm{mg} \cdot 100 \mathrm{~g}^{-1}\right)$, exceto manga (das mais cultivadas) e buriti, pitanga, tucumã e pupunha (pouco cultivadas). Esta mesma observação é feita para proteínas (adequada $>2,5 \mathrm{~g} \cdot 100 \mathrm{~g}^{-1}$ ), exceto para coco (a mais cultivada) e jenipapo, açaí, buriti, cacau, jaca, tamarindo e pupunha (pouco plantadas). A coincidência de cultivo preferencial (IVP) nas residências para coco (rico em proteínas) e manga (vitamina A), além de acerola, caju e

Tabela 2 - Estimativa da concentração de vitaminas e proteínas em frutos comestíveis inventariados na cidade de Boa Vista, Roraima. Todos os valores foram derivados de IBGE (1981), exceto"acerola" e "atemoia", pela Nutrition (2005), "biribá", por Clement (1992) e "dão", pela FAO (2005).

\begin{tabular}{|c|c|c|c|c|c|c|}
\hline $\begin{array}{l}\text { Nome Comum (1) } \\
\text { (Português/Inglês) }\end{array}$ & 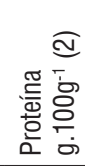 & 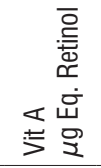 & 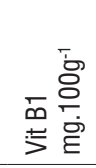 & 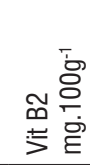 & 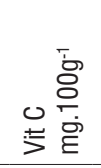 & 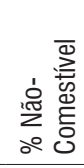 \\
\hline Abacate (Avocado) & 1.8 & 20 & 0.07 & 0.24 & 12 & 34.6 \\
\hline Abiu (Eggfruit) & 2.1 & 46 & 0.02 & 0.02 & 49 & 33.8 \\
\hline Açai (Assai Palm) & 3.8 & - & 0.36 & 0.01 & 9 & 89.6 \\
\hline Acerola (Indian Cherry) & 0.4 & - & - & - & 1678 & - \\
\hline Amora (Red Raspberry) & 1.7 & 2 & 0.03 & 0.06 & 5 & 35.0 \\
\hline Araçá (Brazilian Guava) & 1.5 & 48 & 0.06 & 0.04 & 326 & 17.5 \\
\hline $\begin{array}{l}\text { Araticum (Mountain } \\
\text { Soursop) }\end{array}$ & 0.4 & 50 & 0.04 & 0.07 & 21 & 51.5 \\
\hline Ata (Sugar Apple) & 1.6 & - & 0.11 & 0.15 & 35 & 42.5 \\
\hline Atemoia (Cherimoya) & 1.7 & - & - & - & 12 & - \\
\hline Banana (Banana) (3) & 1.5 & 38 & 0.05 & 0.06 & 17 & 29.7 \\
\hline Biribá (Sweetsop) & - & - & - & - & - & $30-60$ \\
\hline Buriti (Buriti Palm) & 2.6 & 6000 & 0.03 & 0.23 & 26 & 78.7 \\
\hline Cacau (Cacao) & 2.8 & 32 & 0.04 & 0.06 & 10 & 72.2 \\
\hline Caju (Cashew) & 0.8 & 40 & 0.03 & 0.03 & 219 & 19.5 \\
\hline Carambola (Starfruit) & 0.5 & 30 & 0.04 & 0.02 & 35 & 19.0 \\
\hline Coco (Coconut) & 3.5 & - & 0.04 & 0.03 & 4 & 44.0 \\
\hline Cupuaçu (Cupuassu) & 1.7 & 30 & 0.04 & 0.04 & 33 & 49.0 \\
\hline Dão (Indian Jujube) & 1.9 & - & - & - & 58 & - \\
\hline Fruta Pão (Breadfruit) & 1.3 & 2 & 0.08 & 0.06 & 12 & 25.0 \\
\hline
\end{tabular}

goiaba (vitamina C) nos dão pistas de que, mesmo ao acaso, a conjugação destes cultivos caseiros pode ser parte de uma estratégia de sobrevivência (Madaleno, 2000), contribuindo para a suplementação alimentar de populações de baixa renda, como preconizado pela FAO (2004). Populaçôes urbanas de poder aquisitivo menor também usam estes espaços como forma de reunião para confraternização entre amigos e familiares, fazendo da sombra destas árvores um local de lazer e divertimento, fato observado em grandes cidades amazônicas como Belém (Madaleno, 2000) e Manaus (Martins et al., 2003).

Quanto ao número de espécies observadas (riqueza), nossos resultados são semelhantes aos encontrados por Martins et al. (2003) em um bairro popular da cidade de Manaus (Jorge Teixeira), no Amazonas, onde foram computadas 50 espécies de plantas arbóreas distribuídas por 24 famílias botânicas,

Tabela 2 - Continuação

\begin{tabular}{|c|c|c|c|c|c|c|}
\hline Goiaba (Guava) & 0.9 & 26 & 0.04 & 0.04 & 218 & 23.0 \\
\hline Graviola (Soursop) & 1.0 & 2 & 0.07 & 0.05 & 26 & 26.7 \\
\hline Ingá (Inga) & 1.0 & - & 0.04 & 0.06 & 9 & 59.7 \\
\hline Jaca (Jackfruit) & 2.7 & 39 & 0.09 & 0.11 & 9 & 55.0 \\
\hline Jambo (Rose Apple) & 0.8 & 25 & 0.02 & 0.03 & 22 & 19.5 \\
\hline Jenipapo (Genipap) & 5.2 & 30 & 0.04 & 0.04 & 33 & 26.6 \\
\hline Laranja (Orange) (4) & 0.8 & 13 & 0.09 & 0.09 & 59 & 35.8 \\
\hline Lima-Cidra (Citron) & 0.6 & 2 & 0.06 & 0.06 & 32 & 35.2 \\
\hline Limão (Lemon) & 0.6 & 2 & 0.06 & 0.02 & 51 & 54.5 \\
\hline Limão Galego (Lime) & 0.4 & 2 & 0.03 & 0.02 & 40 & 40.0 \\
\hline Mamão (Рapaya) & 0.5 & 37 & 0.03 & 0.03 & 46 & 29.5 \\
\hline Manga (Mango) & 0.5 & 210 & 0.05 & 0.06 & 53 & 39.7 \\
\hline Mirixi (Nance) & 0.9 & 7 & 0.02 & 0.04 & 84 & 46.5 \\
\hline $\begin{array}{l}\text { Pitanga (Surinam } \\
\text { Cherry) }\end{array}$ & 0.8 & 210 & 0.03 & 0.06 & 14 & 33.8 \\
\hline Pitomba (Belizian Genip) & 0.4 & 30 & 0.04 & 0.04 & 33 & 60.0 \\
\hline $\begin{array}{l}\text { Pupunha (Pejibaye } \\
\text { Palm) }\end{array}$ & 2.5 & 1500 & 0.06 & - & 35 & 24.5 \\
\hline Romã (Pomegranate) & 0.8 & - & 0.07 & 0.03 & 8 & 47.3 \\
\hline Siriguela (Red Mombin) & 0.9 & 10 & 0.07 & 0.03 & 45 & 32.3 \\
\hline Tamarindo (Tamarind) & 3.1 & 7 & 0.44 & 0.16 & 6 & 41.6 \\
\hline Tangerina (Tangerine) & 0.7 & 12 & 0.08 & 0.03 & 33 & 29.0 \\
\hline $\begin{array}{l}\text { Taperebá (Yellow } \\
\text { Mombin) }\end{array}$ & 0.8 & 23 & 0.08 & 0.06 & 28 & 49.9 \\
\hline Tucumã (Tucum Palm) (5) & 1.8 & 6000 & 0.01 & 0.02 & 4 & 64.0 \\
\hline
\end{tabular}

(1) exceto "abiurana" e "azeitona roxa"; (2) $100 \mathrm{~g}$ de fruto fresco; (3) média de seis variedades "prata", "São Domingos", "d'água", "figo", "macâa" e "ouro"; (4) variedade "bahia"; (5) derivado da forma geral do Tucum (Astrocaryum spp). 
sendo 43 de frutas comestíveis e 7 não-comestíveis. Naquele estudo, as espécies arbóreo-frutíferas comestíveis foram mais abundantemente distribuídas pelas famílias Anacardiaceae (Mangifera indica-11,4\% e Anacardium occidentale-8,5\%), Sterculiaceae (Theobroma grandiflorum - 8,9\% e T. cacao3,1\%), Myrtaceae (Eugenia malaccensis - 5,6\% e Psidium guajava-5,1\%), Lauraceae (Persea americana-9,1\%) e Arecaceae (Cocos nucifera - 4,7\%). Em Santarém (Pará), WinklerPrins (2002) reconheceu 33 espécies frutíferas (31 arbóreas) nos quintais urbanos, que alimentou a discussão sobre a associação do uso dos recursos naturais dentro do contínuo "rural-urbano". Em ambos os casos, mesmo com diferenças no método de amostragem e quantificação dos recursos arbóreo-frutíferos destas localidades, a preferência por espécies originadas fora da Amazônia é evidente, embora localidades estabelecidas tipicamente em áreas de floresta intercalem com maior frequiência espécies de valor comercial da região como buriti, cupuaçu, pupunha, açaí e cacau.

A tendência do uso dominante de espécies arbóreas frutíferas originadas fora da Amazônia pode se repetir tanto em jardins urbanos como em rurais, talvez como resultado da degradação do conhecimento cultural, científico e comercial das espécies do período pré-colombiano que possuíam algum grau de domesticação na região (Clement, 1990; 1999a; 1999b). Entretanto, Saragoussi et al. (1990), em seu trabalho com três comunidades rurais situadas próximas da cidade de Manaus (AM), indicaram que as espécies "nativas" (derivadas da época pré-colombiana) eram maioria (> 60\%) naqueles quintais, embora espécies "tradicionais" como manga, abacate e citros, tenham sido numericamente mais plantadas pelos agricultores. Estas espécies são populares por razões comuns, tais como por serem tradicionalmente difundidas (troca de material ou presente) e com sementes de fácil transporte sem perda da viabilidade (Gajaseni \& Gasajeni, 1999; Coomes \& Ban, 2004), além de sabor de fácil assimilação humana, fácil cultivo devido a não necessidade de tratos culturais excessivos e alta produtividade.

Para Boa Vista, um dos fatores que também afeta a escolha das espécies é o ambiental. A cidade fica situada em uma área de savana, onde a umidade relativa do ar pode atingir níveis de $30-40 \%$ na estação seca (dezembro-março), e a média de precipitação pluviométrica anual é de $\pm 1.600 \mathrm{~mm} \cdot \mathrm{ano}^{-1}$, ambos os índices considerados baixos em relação a outras localidades da Amazônia (Barbosa, 1997). Portanto, espécies nativas da floresta amazônica (alta umidade e precipitação) teriam dificuldades para se desenvolver em um ambiente diferente, necessitando um investimento de tempo em tratos culturais maior do que o normal para outras espécies mais adaptadas.

Espécies como mirixi (Byrsonima spp.), araçá (Psidium guianense Pers.) e jenipapo (Genipa americana L.), abundantes nas savanas de Roraima, possuem juntas, na média entre os dois bairros, menos de $1 \%$ de IVP. A perda ou a falta de interesse no conhecimento tradicional local, associado aos fatores ambientais, acaba explicando a preferência de uso de algumas espécies introduzidas em relação a outras nativas deste ecossistema de savanas da Amazônia.

\section{CONCLUSÕES}

Nossos resultados sugerem que o cultivo de árvores frutíferas em quintais caseiros de Boa Vista segue um padrão que concentra a escolha em poucas espécies como coco, manga e jambo, não-originárias da Amazônia, mas tradicionalmente consagradas por sua produção de frutos. Em adição, o cultivo de frutíferas arbóreas nos quintais de Boa Vista sugere contribuir com a suplementação alimentar de populaçōes humanas com renda familiar baixa (maior concentração de espécies e indivíduos), além de fornecer opçôes de lazer e sombra.

\section{AGRADECIMENTOS}

À base do INPA em Boa Vista, Roraima, forneceu o suporte logístico do trabalho através do projeto "Ecologia e Manejo dos Recursos Naturais das Savanas de Roraima". Charles Clement (INPA/Manaus) fez críticas e deu sugestôes ao manuscrito original. Dois revisores anônimos contribuíram para texto final.

\section{BIBLIOGRAFIA CITADA}

Albrecht, A.; Kandji, S.T. 2003. Carbon sequestration in tropical agroforestry systems. Agriculture, Ecosystems and Environment, 99: 15-27.

Barbosa, R.I. 1997. Distribuição das chuvas em Roraima. In: Barbosa, R.I.; Ferreira, E.J.G.; Castellon, E.G. (eds.), Homem, Ambiente e Ecologia no Estado de Roraima. Instituto Nacional de Pesquisas da Amazônia, Manaus. pp. 325-335.

Balée, W. 1989. The culture of Amazonian forests. In: Posey, D.A.; Balée, W. (eds.), Resource Management in Amazonia: Indigenous and Folk Strategies. New York Botanical Garden (Advances in Economic Botany 7), New York. pp. 1-21.

Blanckaert, I.; Swennen, R.L.; Paredes Flores, M.; Rosas López, R.; Lira Saade, R. 2004. Floristic composition, plant uses and management practices in homegardens of San Rafael Coxcatlán, Valley of Tehuacán-Cuicatlán, Mexico. Journal of Arid Environments, 57: 39-62.

Cavalcante, P.B. 1991. Frutas comestiveis da Amazônia. Museu Paraense Emílio Goeldi (Coleção Adolfo Ducke), Belém. 279pp.

Clement, C.R. 1990. Origin, domestication and genetic conservation of Amazonian fruit tree species. In: Posey, D.A.; Overal, W.L. (org.), Ethnobiology: Implications and Applications, Proceedings of the First International Congress of Ethnobiology (v. 1). Museu Paraense Emílio Goeldi, Belém. pp. 249-263. 
Clement, C.R. 1992. Frutas da Amazônia. Ciência Hoje, 14(83): $28-37$.

Clement, C.R. 1999a. 1492 and the loss of the Amazonian crop genetic resources. I. The relation between domestication and human population decline. Economic Botany, 53(2): 188-202.

Clement, C.R. 1999b. 1492 and the loss of the Amazonian crop genetic resources. II. Crop biogeography at contact. Economic Botany, 53(2): 203-216.

Clement, C.R.; Noda, H.; Noda, S.N.; Martins, A.L.U.; Silva, G.C. 2001. Recursos frutícolas na várzea e na terra firme em onze comunidades rurais do Alto Solimões, Amazonas, Brasil. Acta Amazonica, 31(3): 521-527.

Coomes, O.T.; Ban, N. 2004. Cultivated plant species diversity in home gardens of an Amazonian peasant village in northeastern Peru. Economic Botany, 58(3): 420-434.

FAO 2004. Household food security and community nutrition. Food and Agriculture Organization of the United Nations, Roma. Disponível em: http://www.fao.org/es/ESN/nutrition/ household_gardens_en.stm. Acesso em: dezembro de 2004 .

FAO 2005. Food composition table for use in Africa: Vegetables andvegetable products (Composition in Terms of 100 Grams Edible Portion). Food and Agriculture Organization of the United Nations, Roma. Disponível em: http:/www.fao.org/docrep/003/ X6877E/X6877E13.htm. Acesso em: março de 2005.

Fernandes, E.C.M.; Nair, P.K.R. 1986. An evaluation of the structure and function of tropical homegardens. Agricultural Systems, 21: 279-310.

Gajaseni, J.; Gasajeni, N. 1999. Ecological rationalities of the traditional homegarden system in the Chao Phraya Basin, Thailand. Agroforestry Systems, 46: 3-23.

IBGE 1981. Estudo Nacional de Despesa Familiar (ENDEF) - Tabelas de Composição de Alimentos (2a ed.). Instituto Brasileiro de Geografia e Estatística, Rio de Janeiro. 213pp.

IBGE 2002. Censo Demográfico do Brasil 2000. Instituto Brasileiro de Geografia e Estatística, Rio de Janeiro. Disponível em http:// www.ibge.gov.br. Acessado em: dezembro de 2004.

IBGE 2004. Situação demográfica dos bairros de Boa Vista. Instituto Brasileiro de Geografia e Estatística, Delegacia Regional de Roraima, Boa Vista. 1p. (Relatório Técnico produzido a partir dos dados originais do Censo 2000 do IBGE).

Kent, M.; Coker, P. 1994. Vegetation Description and Analysis - A Practical Approach. Wiley, Chichester. 363pp.

Kimber, C. 1966. Dooryard gardens of Martinique. Yearbook of the Pacific Coast Geographers, 28: 97-118.

Madaleno, I. 2000. Urban agriculture in Belém, Brazil. Cities, 17(1): 73-77.

Magurran, A.E. 1988. Ecological diversity and its measurement. Croom-Helm, Londres. 179pp.

Martin, G.J. 1995. Ethnobotany: a methods manual. Chapman \& Hall, Londres. 268p.

Martins, A.L.U. 1998. Quintais urbanos em Manaus: organização, espaço e recursos naturais. Dissertação de Mestrado, UFAM, Manaus.
Martins, A.L.U.; Noda. H.; Noda, S.N. 2003. Quintais Urbanos de Manaus. In: Oliveira, J.A.; Alecrim, J.D.; Gasnier, T.R.J. (orgs.), Cidade de Manaus: visóes interdisciplinares. Editora da Universidade do Amazonas (EDUA), Manaus. pp. 207-244.

McConnell, D.J. 1992. The Forest Garden Farms of Kandy, Sri Lanka. Food and Agriculture Organization of the United Nations, Roma (FAO Farm Systems Management, Series 3).

Miranda, I.S. 1998. Flora, fisionomia e estrutura das savanas de Roraima, Brasil. Tese de Doutorado. Instituto Nacional de Pesquisas da Amazônia (INPA) / Fundação Universidade do Amazonas, Manaus. 186pp.

Miranda, I.P.A.; Rabelo, A.; Bueno, C.R.; Barbosa, E.M.; Ribeiro, M.N.S. 2001. Frutos de palmeiras da Amazônia. Instituto Nacional de Pesquisas da Amazônia (INPA), Manaus. 120pp.

MS 2002. Alimentos regionais brasileiros. Ministério da Saúde, Diretoria Técnica da Coordenação Geral da Política de Alimentação e Nutrição, Brasília. Série Comunicação e Educação em Saúde, 21. 140pp.

Nair, P.K.R. 1993. An Introduction to Agroforestry. Kluwer Academic Publishers, Dordrecht. 499pp.

Nutrition 2005. Nutrition Facts and Food Composition. Disponível em http://www.nutritionanalyser.com/food_composition. Acesso em: março de 2005.

Pahlen, A. von der; Kerr, W.E.; Paiva, W.O.; Rahman, F.; Yuyama, K.; Pahlen, E. von der; Noda, H. 1979. Introdução à horticultura e fruticultura no Amazonas. Instituto Nacional de Pesquisas da Amazônia (INPA) / Superintendência da Zona Franca de Manaus (SUFRAMA) / Imprensa Oficial do Estado do Amazonas, Manaus. 140pp.

Rodrigues, R.M. 1989. A flora da Amazônia. Centro de Estudos Jurídicos do Pará, Belém. 462pp.

Sanaiotti, T.M. 1996. The woody flora and soils of seven Brazilian Amazonian dry savanna areas. Tese de Doutorado. University of Stirling, Stirling. 145pp.

Saragoussi, M.; Martel, J.H.I.; Ribeiro, G.A. 1990. Comparação na composição de quintais de três localidades de terra firme no estado do Amazonas. In: Posey, D.A.; Overal, W.L. (org.), Ethnobiology: Implications and Applications, Proceedings of the First International Congress of Ethnobiology (v. 1). Museu Paraense Emílio Goeldi, Belém. pp. 295-303.

Silva, M.F.; Lisboa, P.L.B.; Lisboa, R.C.L. 1977. Nomes vulgares de plantas amazônicas. Instituto Nacional de Pesquisas da Amazônia (INPA), Manaus. 222pp.

Shanley, P.; Cymerys, M.; Galvão, J. 1998. Frutiferas da mata na vida Amazônica. Editora Supercores, Belém. 125pp.

WinklerPrins, A.M.G.A. 2002. House-lot gardens in Santarém, Pará, Brazil: Linking rural with urban. Urban Ecosystems, 6(1-2): 43-65.

Recebido em 09/03/2006

Aceito em 04/07/2007 